\title{
The influence of the extracellular domains on the dynamic behavior of membrane proteins
}

\author{
Linda Wedemann ${ }^{1,3 \#}$, Cenk Onur Gurdap ${ }^{1, \#}$, Taras Sych $^{1}$ and Erdinc Sezgin ${ }^{1,2}$
}

\author{
${ }^{1}$ Science for Life Laboratory, Department of Women's and Children's Health, Karolinska \\ Institutet, 17165 Solna, Sweden;
}

2 MRC Human Immunology Unit, MRC Weatherall Institute of Molecular Medicine, University of Oxford, Oxford OX3 9DS, U.K.

${ }^{3}$ Current Address: Centre for Structural Systems Biology, Hamburg, Germany; Hannover Medical School, Institute of Virology, Hannover, Germany; Cluster of Excellence RESIST (EXC 2155), Hannover Medical School, Hannover, Germany; Heinrich Pette Institute Leibniz-Institute for Experimental Virology, Hamburg, Germany

\#contributed equally

correspondence: erdinc.sezgin@ki.se 


\begin{abstract}
The dynamic behavior of the plasma membrane proteins mediates various cellular processes, such as cell-cell interactions, transmembrane transport and signaling. It is widely accepted that the dynamics of the membrane proteins is determined either by the interactions of the transmembrane domain with the surrounding lipids or by the interaction of the intracellular domain with cytosolic components such as cortical actin. However, the impact of the extracellular domains (ECDs) on the dynamics of membrane proteins is rather unexplored. Here, we investigate how the ECD size influences protein dynamics in lipid bilayer. We reconstitute ECDs of different molecular weights and heights in model membrane systems and analyze ECD-driven protein sorting in lipid domains as well as protein mobility. We observe that increasing the ECD size leads to a decrease in ordered domain partitioning as well as diffusivity. Our data suggests a critical role of the ECDs on membrane protein behavior in the plasma membrane and paves the way to a more complete understanding of membrane protein dynamics that includes interaction with the extracellular matrix and glycocalyx in health and disease.
\end{abstract}

Keywords: membrane proteins, extracellular domain, model membranes, GUV, GPMV, NiNTA, phase separation, diffusion, FCS 


\section{INTRODUCTION}

The plasma membrane (PM) is a complex and dynamically heterogeneous system consisting of a lipid bilayer populated with various lipids and membrane proteins (MPs). About $40 \%$ of the human proteome is membrane-associated proteins ${ }^{1}$ that are either integrally embedded into the PM or peripherally associated by the interactions with the headgroups of the inner leaflet lipids. Integral MPs are embedded in the membrane via one or multiple transmembrane domain(s) (TMD), with extramembraneous hydrophilic domains extending into the cytosol (intracellular domain, ICD) or extracellular environment (extracellular domain, ECD). Integral proteins are mobile within the membrane lateral plane, allowing them to assemble into complexes for various molecular interactions ${ }^{2,3}$. These molecular interactions lead to a highly heterogenous architecture for the $\mathrm{PM}^{4}$.

Liquid-liquid phase separation (LLPS) is a subset of PM heterogeneity caused by lipidlipid and lipid-protein interactions. Model membranes are widely used as a synthetic proxy of such heterogeneity in the $\mathrm{PM}^{5}$. In these simple model membrane systems, saturated lipids form a liquid-ordered phase ( $\left.L_{0}\right)$ with the help of cholesterol, and unsaturated lipids form a liquid-disordered phase $\left(L_{d}\right)$. Different artificial or cell-derived model systems are available to study this phase separation ${ }^{5}$. Giant unilamellar vesicles (GUVs) and giant plasma membrane vesicles (GPMVs) are commonly used cell-sized spherical free-standing lipid bilayers. While GPMVs are cell-derived and thereby inherently more complex in lipid and protein compositions, GUVs are artificial systems with finely controlled composition. Both systems could display the co-existing phases to mimic the lateral heterogeneity of the PM. Proteins were shown to partition into one of these phases preferentially. Such selective protein partitioning can induce lipid environment-dependent conformational change and promote lipid-driven protein association crucial for protein function, such as immune cell function and viral dynamics ${ }^{6-9}$. Recent studies showed that length, lipid accessible surface area, and the addition of fatty-acid-like molecules such as palmitoylation at the TMD can influence protein partitioning in the membranes ${ }^{10-12}$. The glycosylphosphatidylinositol (GPI)-anchored proteins partition based on the structure of their protein anchors ${ }^{13}$. Critical factors in phase separation and protein partitioning are reviewed in detail in refs ${ }^{4,14}$. While there is extensive research on influence of TMD properties on phase partitioning of proteins ${ }^{12}$, the role of ECDs on partitioning is largely unexplored. Some 
of the earlier work performed using biochemical methods such as detergent resistance suggested a non-existing role of ECDs in membrane domain partitioning of proteins such as CD4 ${ }^{15}$. In recent years, however, the role of ECDs on the membrane structure and dynamics have been addressed using state-of-the-art methods. For example, it was shown that protein assemblies at the extracellular side of the membrane induce membrane reorganization such as membrane bending 16, tube formation 17, concomitant polymerization ${ }^{18}$, or domain dissolution ${ }^{19}$. Moreover, ECD size has been one of the major players in immune synapse formation ${ }^{20}$, continuously being studied in the context of lipid remodeling ${ }^{21}$. This evidence necessitates a thorough study on the effect of ECDs on the partitioning of the MPs.

To exhibit their function, MPs need to be dynamic since their lateral mobility allows them to interact with other proteins and thereby form complexes. Therefore, measuring the lateral mobility of proteins is crucial to gain a mechanistic understanding of their function. Influenced by the compositional complexity, the dynamics of MPs are not only based on Brownian free motion but rather more complex, mediated by lipid domains, protein-protein interactions, and cytoskeletal elements 22-27. According to the established Saffman and Delbrück model, when a molecule is anchored to the membrane, its diffusion depends on membrane viscosity, lipid thickness, and weakly on the radius of the TMD ${ }^{28}$. There have been several corrections to this model ${ }^{29-35}$; however, they are mainly focused on the impact of lipid environment, TMD and ICD of the proteins on lateral diffusion, with exception of GPI-anchored proteins where some hints of influence of protein size on diffusion were observed, ${ }^{36,37}$. Most recently, the computational simulations ${ }^{38}$ and experimental methods ${ }^{39}$ have predicted that the diffusion of MPs might also be influenced by the ECDs.

In this work, we reconstituted hexahistidine (6xHis)-tagged ECDs of different sizes in model membranes functionalized with Ni-NTA lipids. In this setting, TMDs and ICDs are absent, hence the impact of ECDs on the diffusion can be directly elucidated. To monitor the phase partitioning of ECDs, we used phase-separated GUVs and, interestingly, we found that protein sorting to $L_{o}$ decreases with increasing ECD sizes. Furthermore, we studied ECD mobility in GUVs of different compositions and GPMVs isolated from $\mathrm{CHO}$ cells. We found that protein mobility also decreases as the ECD size increases. Our data suggests a critical role of the ECDs on membrane protein behavior in the plasma membrane and paves the way to a more complete 
understanding of membrane protein dynamics that includes interaction with the extracellular matrix and glycocalyx in health and disease.

\section{MATERIALS AND METHODS}

Details on all materials and methods are in Supplementary Document.

\section{RESULTS AND DISCUSSIONS}

We employed GUVs and GPMVs as model systems to mimic cellular membrane surfaces and study protein behavior. The main advantage of GUVs is the finely controlled lipid composition while that of GPMVs is their near-native composition. We studied protein phase partitioning in phase separated GUVs, and protein diffusion in homogeneous GUVs and GPMVs.

\section{Partitioning of saturated and unsaturated nickelated lipids in phase separated vesicles}

To study the role of ECD size on protein partitioning, we first prepared phase-separated GUVs (DOPC/SM/Chol, 2:2:1) with $1 \mathrm{~mol} \%$ of nickelated lipid that can directly bind to 6xHis-tagged ECDs (Fig. 1A). To visualize phase separation, we used AbStR-DPPE that partitions preferentially to $L_{d}($ Fig. $1 A, B)$. To evaluate the partitioning of proteins in both ordered and disordered phases, we used two different nickelated lipids: unsaturated 18:1/18:1 (we will refer to it as di-oleyl (DO) Ni-NTA) and saturated 16:0/16:0 Ni-NTA (we will refer to it as di-palmitoyl (DP) Ni-NTA). To recognize and visualize Ni-NTA, we employed Alexa 488-6xHis (A488-6xHis). We observed that in GUVs with DO Ni-NTA, A488-6xHis almost exclusively binds to $\mathrm{Ld}_{d}$ and in GUVs with DP Ni-NTA, it is preferentially associated with Lo (Fig.1B) as expected.

Artificial phase domains in GUVs display high contrast in membrane order between Lo and $L_{d}$ domains. Alternatively, cell-derived GPMVs present more complex domains with less dramatic difference in membrane order. To evaluate the partitioning of these Ni-NTA lipids in a more natural system, we incorporated nickelated lipids into GPMVs (Fig. 1C). However, incorporating Ni-NTA lipids to GPMVs is rather challenging compared to GUVs presumably due to the charged surface of GPMVs (as the negatively charged lipids flip from the inner leaflet). Another possible reason might be the high DTT concentration used to form phase-separated GPMVs, which possibly reacts with nickel and reduces it. Despite these challenges, we obtained a small 
number of phase-separated GPMVs with nickelated lipids attached to the proteins (Fig. 1D) and confirmed that DO Ni-NTA partitions to the disordered domains whereas DP Ni-NTA prefers the ordered domains.

Since GUVs with Ni-NTA lipids were significantly easier to prepare, we continued with GUVs for the rest of the partitioning experiments, where we evaluated the role of ECD size on partitioning. For this purpose, we selected ECDs of the proteins that are mainly involved in immune signaling: CD59, CD2, ICAM-1, CD45, and CD22 whereas A4886xHis was used as a control (Fig. 1E). Estimated sizes of these proteins are shown in Fig.1E, and the number of aminoacids in these peptides are shown in Supp. Table 1.

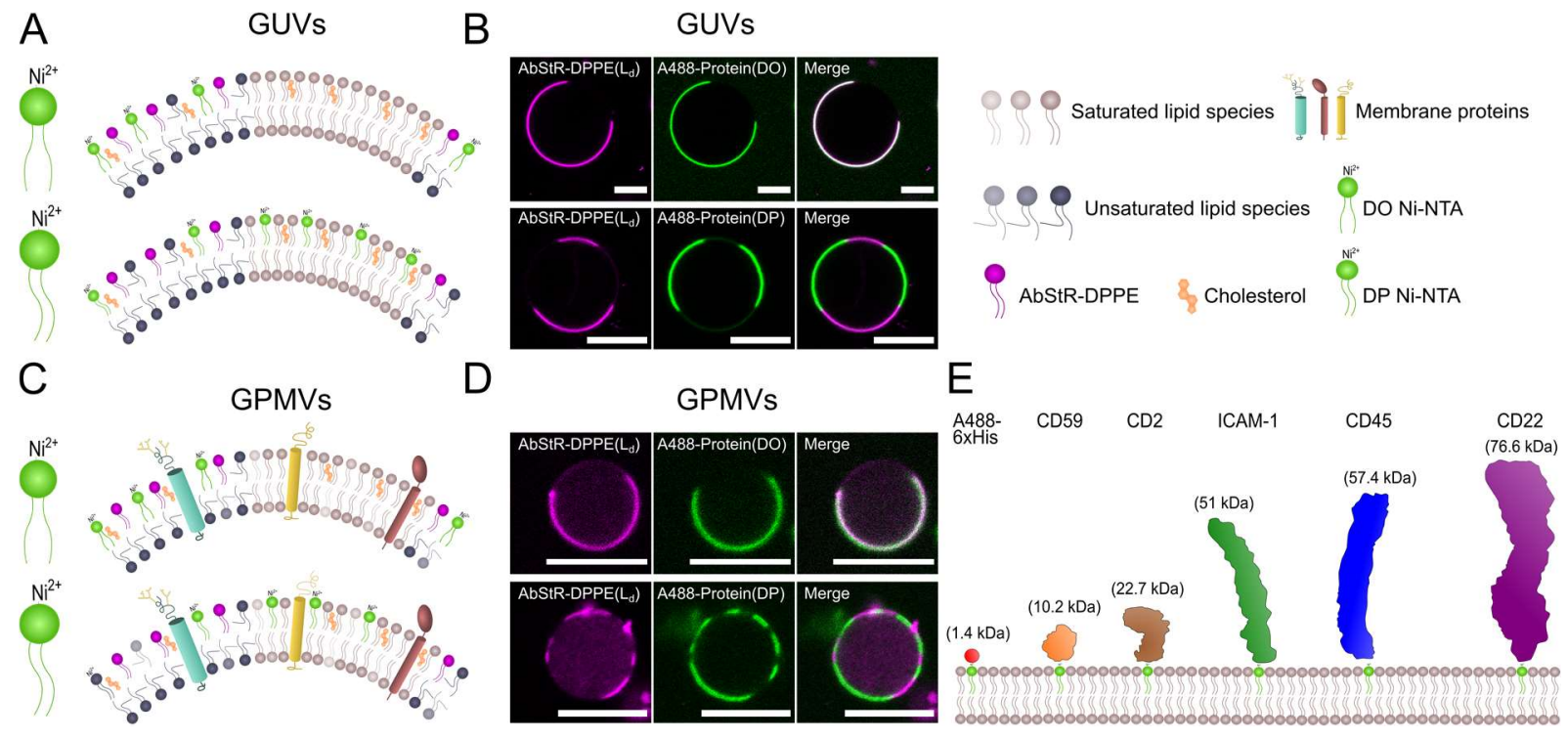

Fig. 1. Ni-NTA lipid constituted biomimetic membranes with His-tagged proteins. (A) Cartoon of phase-separated GUVs incorporated with DO and DP Ni-NTA and (B) confocal microscopy images. GUVs are composed of the ternary mixture of DOPC/SM/Chol (2:2:1). (C) Cartoon of phase-separated GPMVs incorporated with DO and DP Ni-NTA and (D) confocal microscopy images. GPMVs are extracted from HeLa cells. AbStR-DPPE (magenta) and DO Ni-NTA incorporate preferentially in disordered phase, whereas DP Ni-NTA prefers ordered phase. Scale bars are $10 \mu \mathrm{m}$. (E) Proteins of interest used in the study (sizes are representative based on the structures).

\section{ECD size is a determinant of the protein partitioning}

Although the ECDs often do not directly interact with the PM, they might still play a role in protein partitioning given the recent evidence on the impact of extracellular protein organization on membrane domains ${ }^{16-19}$. Earlier work using detergent resistance suggested certain sequences in ECDs can be ordered domain targeting sequences 
40,41. However, the size of the ECDs has not been evaluated in this context. Therefore, we set out to evaluate whether ECD size affects protein partitioning in phase-separated GUVs. To investigate this, ECDs of different sizes (Fig. 1E) were incorporated into phase-separated GUVs. (Fig. 2). The fluorescence intensity of the two peaks in the line profile across the GUV image (A488-protein and AbStR-DPPE) was used to calculate the percentage of $L_{\circ}$ partitioning (Fig. 2A).

To examine whether the concentration of the proteins on the surface affects partitioning, we first performed a control experiment with varying A488-6xHis concentrations. The result indicates that there is no effect of the concentration of A488$6 x \mathrm{His}$ on its partitioning preference within the investigated concentration range (Fig. 2B). After this, we started investigating the effect of ECD size on protein partitioning. As mentioned earlier, the partitioning preference of proteins majorly depends on the structure of nickelated lipid. In GUVs with DO Ni-NTA, proteins partitioned preferentially to $L_{d}$, whereas in GUVs with DP Ni-NTA to Lo. Interestingly, in both systems, Lo partitioning decreased as the molecular size increased (Fig. 2C-D). Curiously, CD22 deviated from this trend in GUVs with DP Ni-NTA: Lo preference of CD22 was even higher than the two smallest ECDs despite its ECD size (Fig. 2D). The reason could be the potential formation of protein clusters of CD22. These findings imply that ECD size might be one of the determining factors for protein partitioning, and larger proteins might be less abundant in ordered domains. These proteins might need more specific driving forces (such as TMD, acylation, clustering, interactions) to reside in the ordered domains. 


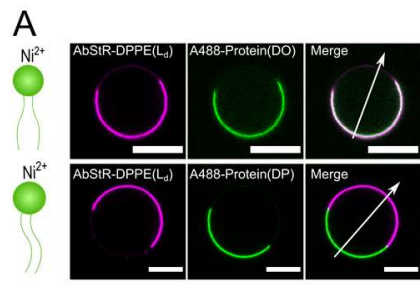

$\% L_{0}=\frac{I_{L_{0}}}{I_{L_{o}}+I_{L_{d}}}$

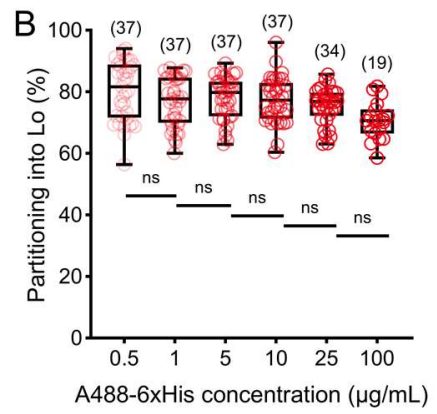

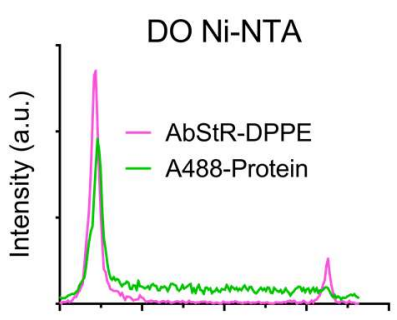

Distance $(\mu \mathrm{m})$

DO Ni-NTA

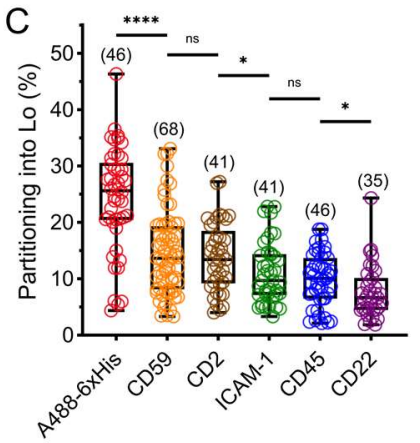

DP Ni-NTA

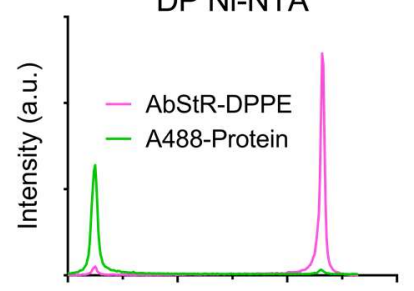

Distance $(\mu \mathrm{m})$

DP Ni-NTA

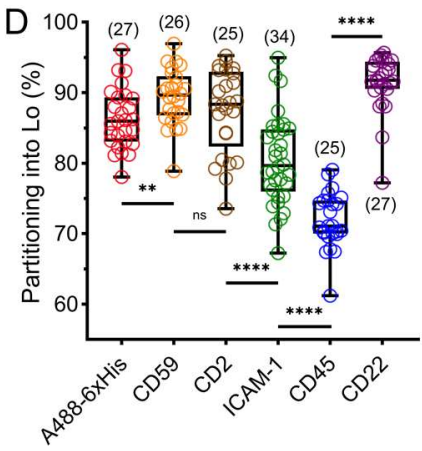

Fig. 2. ECD size plays a role in protein partitioning in GUVs. (A) Line profile of fluorescence intensity across the GUVs indicated by the white arrow in the confocal images were used to calculate Lo percentage with the equation shown below the images. Scale bars are $10 \mu \mathrm{m}$. (B) Lo partitioning coefficient with different concentrations of A488-6xHis (significance evaluated by non-parameterized ANOVA). (C) Lo partitioning of different ECDs attached to DO Ni-NTA in GUVs. Student's t-test (twotailed, non-parameterized) was used to determine significance $\left({ }^{* * *} \mathrm{P}<0.0001\right.$; CD2 vs. ICAM1: $P=0.0393$; $C D 45$ vs. CD22: $P=0.024$ ). (D) $L_{0}$ partitioning of the different $E C D s$ attached to the DP NiNTA lipid in GUVs. Student's t-test (two-tailed, non-parameterized) was used to determine significance $\left({ }^{* * *} \mathrm{P}<0.0001 ;{ }^{* *} \mathrm{P}=0.005\right)$. Error bars represent the standard deviation. The numbers of data points obtained from experiments are indicated on the graphs in parentheses.

\section{$E C D$ size is a determinant of the protein diffusion}

There is still a controversy in the structural determinants of MP mobility. From the very first diffusion model ${ }^{28}$ to the following modifications ${ }^{31-35}$, none of them explicitly takes ECD size into account. There is a consensus in the field that the diffusion of protein in the PM is determined by the TMD, the lipid environment and their attachment to the membrane. To investigate the effect of ECDs on protein dynamics in the membrane, here, we measured the diffusion coefficients of proteins using FCS where fluorescence intensity fluctuations were used to obtain information about molecular diffusion (Fig. $3 A$ ). We first tested how the diffusion of ECDs was affected by their sizes in GUVs of different compositions. For this purpose, we measured the diffusion of ECDs in POPC 
(Fig. 3B), POPC/Chol (1:1) (Fig. 3C), and DPPC/Chol (1:1) (Fig. 3D) membranes doped with $1 \mathrm{~mol} \%$ DO Ni-NTA. Membrane composed of POPC is relatively fluid and becomes more rigid when combined with cholesterol (POPC:Chol). Finally, DPPC/Chol presents the most rigid membrane among the compositions we used herein. In all cases, we see a reciprocal relationship between the mobility and the ECD size, that is, increase in the size of the protein leads to slower diffusion. The mobility measurement displays no change in diffusion of lipid analogue AbStR-DPPE in GUVs with ECDs of different sizes (Supp. Fig. 1A), confirming that the membrane physical parameters (e.g. fluidity) and the experimental conditions (e.g., temperature fluctuations), did not cause the differences in diffusion for different ECDs. To visualize the effect of lipid environment on the changes of diffusion for different ECDs, we plotted the fold change in diffusion for different lipid compositions compared to the diffusion in POPC membrane (Fig. 3E). This comparison shows that the ECD size affects the diffusion similarly in different lipid environments. Only exception was again CD22 which showed a higher slow-down in POPC vesicles compared to POPC/Chol or DPPC/Chol vesicles (Fig. $3 \mathrm{E}$ ). This can be due to its non-monomeric organization in the membrane. 


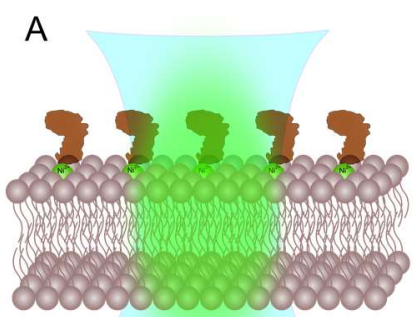

B
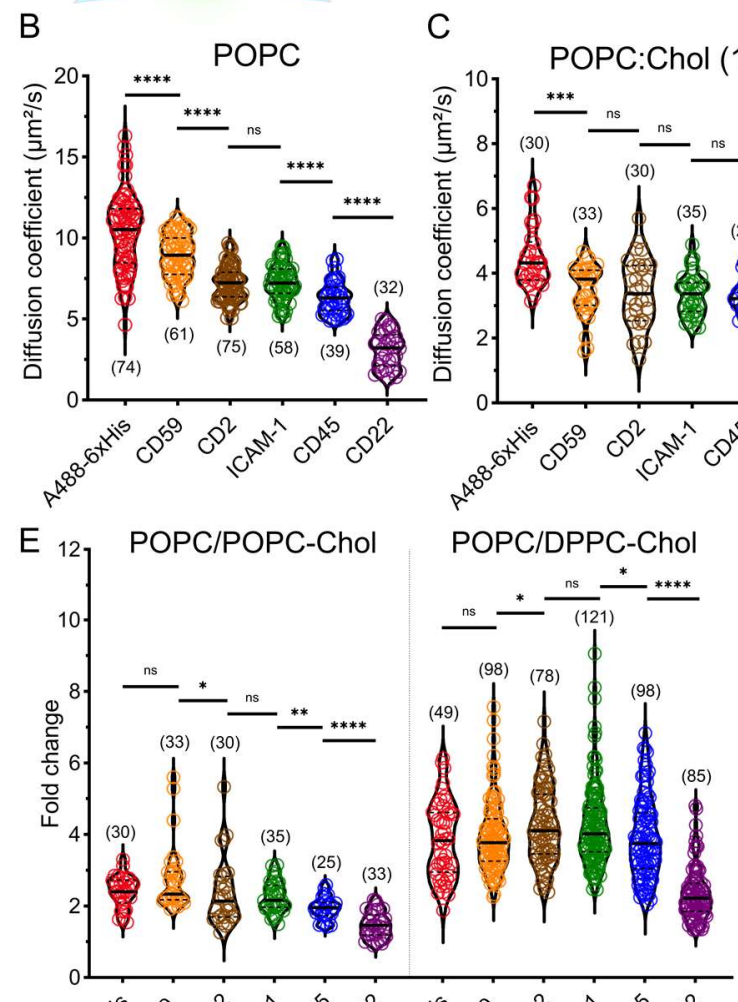

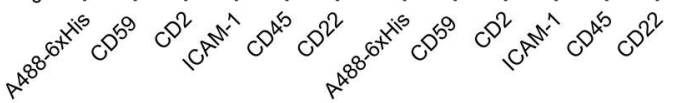

C

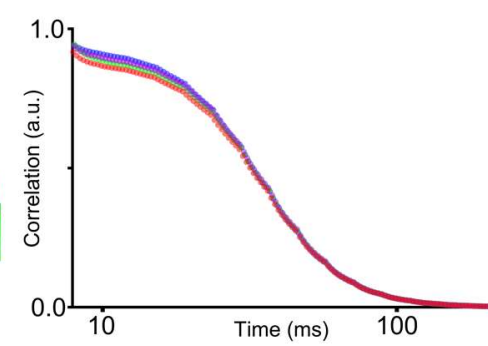
POPC:Chol (1:1)

$\mathrm{D}$

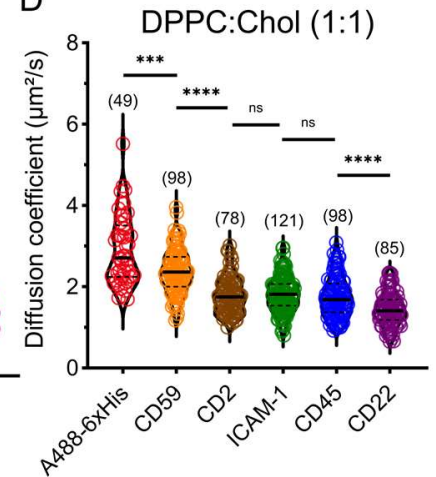

F

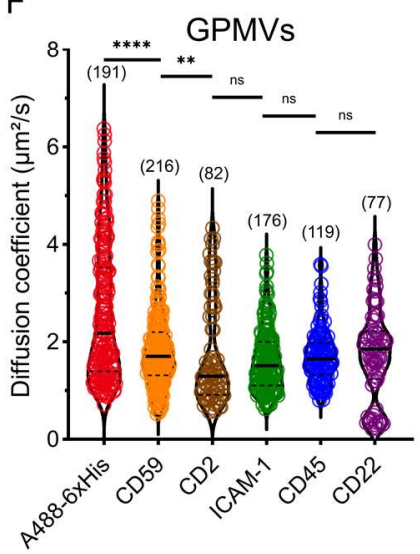

Fig. 3. ECD size determines protein diffusion in GUVs and GPMVs. (A) Illustration of the FCS experiment. Different colors in correlation graph indicate data of multiple repetitions of diffusion of the same protein which indicates high reproducibility. (B-D) Diffusion coefficients of the different ECDs in GUVs with (B) POPC, (C) with POPC:Chol (1:1), and (D) with DPPC/Chol (1:1). (E) The fold change comparison of proteins (diffusion in POPC is divided by diffusion in POPC/Chol and DPPC/Chol, respectively). Student's t-test (two-tailed, non-parameterized) was used to determine significance $\left({ }^{* * *} \mathrm{P}<0.0001\right.$; POPC/POPC-Chol: ${ }^{*} \mathrm{P}=0.0197,{ }^{* *} \mathrm{P}=0.0045$; POPC/DPPC-Chol: CD59 vs. CD2: $P=0.0376$, ICAM-1 vs. CD45: $P=0.021)$. ( $F)$ Diffusion of the different ECDs in GPMVs. Student's t-test (two-tailed, non-parameterized) was used to determine significance ${ }^{* * * *} \mathrm{P}<0.0001 ; \mathrm{POPC} / \mathrm{Chol}$ : ${ }^{* \star *} \mathrm{P}=0.0002$; DPPC/Chol: ${ }^{* *} \mathrm{P}=0.0006$; GPMVs: $\left.{ }^{* *} \mathrm{P}=0.0054\right)$. Error bars represent the standard deviation. The numbers of data points obtained from experiments are indicated on the graphs in parentheses. 
Since cellular PM is a more crowded and complex environment than the GUV system we used, the size effect can be diluted in this crowded environment. To test this in more physiological model membranes, we next performed the experiment in GPMVs. The variation in membrane compositions between individual GPMVs leads to larger variability of the diffusion coefficient, therefore the data spread in graphs is larger. Despite the large standard deviations, diffusion of proteins in GPMVs generally resembles the trend in GUVs with certain exceptions. The diffusion coefficient decreases with an increase in ECD size for the first three small proteins, where the size differences between the proteins are the largest (Fig. 3F). For relatively larger proteins with small differences in size (e.g., ICAM-1 and CD45), diffusion did not differ drastically. For CD22, we observed two populations in diffusion: first population is similar to other large proteins while the second population is significantly slower which might support the clustering hypothesis we discussed before. AbStR-DPPE shows no significant change in diffusion with ECDs of different sizes, confirming that the observed differences are indeed due to ECD size. This data means that even in more physiological settings, the size of ECDs is crucial, but in contrast to GUVs, small differences in size can be masked by other factors in the native membranes such as molecular crowding. The mobility measurement of lipid analog

\section{CONCLUSIONS}

In this study, we investigated largely overlooked role of ECD size on MP dynamics. Our results show a decrease both in partitioning into ordered domains and in diffusion coefficient with the increase in ECD size. In the light of our data, we suggest that ECD is a necessary component for understanding the protein behavior in the PM. It is, however, essential to acknowledge the limitations of our study. The direct translatability from model membrane systems to the native PM requires caution. The absence of other membrane proteins (i.e., absence of protein-protein interactions), the cortical actin cytoskeleton and the extracellular networks (i.e., lack of crowding) might hinder direct translatability of GUV experiments. Here, cell-derived GPMVs fill the gap to a certain extent but they are also model membrane systems with several shortcomings. In addition, all ECDs but CD59 originate from integral proteins, hence, we refrain from drawing conclusions on the full-length proteins. 
bioRxiv preprint doi: https://doi. org/10.1101/2021.11.15.468619; this version posted November 16, 2021. The copyright holder for this preprint (which was not certified by peer review) is the author/funder, who has granted bioRxiv a license to display the preprint in perpetuity. It is made available under aCC-BY-NC 4.0 International license.

Despite the aforementioned limitations, our system provides a clean and controllable environment to evaluate how ECD size influences the dynamic behavior of the proteins independent of their TMDs or ICDs. We believe that this work will pave the way for further experiments and simulations which will eventually lead to new models for protein dynamics in the PM.

\section{ACKNOWLEDGMENTS}

We thank Hannes Feyrer for his feedback on visuals, SciLifeLab Advanced Light Microscopy facility and National Microscopy Infrastructure (VR-RFI 2016-00968) for their support on imaging. COG was supported by a SciLifeLab summer internship (C2021-0466), ES is supported by grants from Swedish Research Council Starting Grant (2020-02682), from Karolinska Institutet and from the SciLifeLab National COVID-19 Research Program, financed by the Knut and Alice Wallenberg Foundation.

\section{REFERENCES}

(1) Fagerberg, L.; Jonasson, K.; von Heijne, G.; Uhlén, M.; Berglund, L. Prediction of the Human Membrane Proteome. Proteomics 2010, 10 (6), 1141-1149. https://doi.org/10.1002/pmic.200900258.

(2) Niemelä, P. S.; Miettinen, M. S.; Monticelli, L.; Hammaren, H.; Bjelkmar, P.; Murtola, T.; Lindahl, E.; Vattulainen, I. Membrane Proteins Diffuse as Dynamic Complexes with Lipids. J Am Chem Soc 2010, 132 (22), 7574-7575. https://doi.org/10.1021/ja101481b.

(3) Saka, S. K.; Honigmann, A.; Eggeling, C.; Hell, S. W.; Lang, T.; Rizzoli, S. O. MultiProtein Assemblies Underlie the Mesoscale Organization of the Plasma Membrane. Nature Communications 2014, 5. https://doi.org/10.1038/ncomms5509.

(4) Sezgin, E.; Levental, I.; Mayor, S.; Eggeling, C. The Mystery of Membrane Organization: Composition, Regulation and Physiological Relevance of Lipid Rafts. Nat Rev Mol Cell Biol 2017, 18 (6), 361-374. https://doi.org/10.1038/nrm.2017.16.

(5) Sych, T.; Gurdap, C. O.; Wedemann, L.; Sezgin, E. How Does Liquid-Liquid Phase Separation in Model Membranes Reflect Cell Membrane Heterogeneity? Membranes 2021, 11 (5), 323. https://doi.org/10.3390/membranes 11050323.

(6) Lorent, J. H.; Levental, I. Structural Determinants of Protein Partitioning into Ordered Membrane Domains and Lipid Rafts. Chemistry and Physics of Lipids 2015, 192, 23 32. https://doi.org/10.1016/j.chemphyslip.2015.07.022.

(7) Polley, A.; Orlowski, A.; Danne, R.; Gurtovenko, A. A.; Bernardino de la Serna, J.; Eggeling, C.; Davis, S. J.; Rog, T.; Vattulainen, I. Glycosylation and Lipids Working in Concert Direct CD2 Ectodomain Orientation and Presentation. The journal of physical chemistry letters 2017, 1060-1066. https://doi.org/10.1021/acs.jpclett.6b02824. 
(8) Stone, M. B.; Shelby, S. A.; Núñez, M. F.; Wisser, K.; Veatch, S. L. Protein Sorting by Lipid Phase-like Domains Supports Emergent Signaling Function in B Lymphocyte Plasma Membranes. eLife 2017, 6, e19891. https://doi.org/10.7554/eLife.19891.

(9) Yang, S.-T.; Kreutzberger, A. J. B.; Kiessling, V.; Ganser-Pornillos, B. K.; White, J. M.; Tamm, L. K. HIV Virions Sense Plasma Membrane Heterogeneity for Cell Entry. Sci Adv 2017, 3 (6), e1700338. https://doi.org/10.1126/sciadv.1700338.

(10) Sharpe, H. J.; Stevens, T. J.; Munro, S. A Comprehensive Comparison of Transmembrane Domains Reveals Organelle-Specific Properties. Cell 2010, 142 (1), 158-169. https://doi.org/10.1016/j.cell.2010.05.037.

(11) Diaz-Rohrer, B. B.; Levental, K. R.; Simons, K.; Levental, I. Membrane Raft Association Is a Determinant of Plasma Membrane Localization. PNAS 2014, 111 (23), 8500-8505. https://doi.org/10.1073/pnas.1404582111.

(12) Lorent, J. H.; Diaz-Rohrer, B.; Lin, X.; Spring, K.; Gorfe, A. A.; Levental, K. R.; Levental, I. Structural Determinants and Functional Consequences of Protein Affinity for Membrane Rafts. Nat Commun 2017, 8 (1), 1219. https://doi.org/10.1038/s41467017-01328-3.

(13) Nalivaeva, N. N.; Turner, A. J. Lipid Anchors to Proteins. In Handbook of Neurochemistry and Molecular Neurobiology: Neural Lipids; Lajtha, A., Tettamanti, G., Goracci, G., Eds.; Springer US: Boston, MA, 2009; pp 353-372. https://doi.org/10.1007/978-0-387-30378-9_14.

(14) van Deventer, S.; Arp, A. B.; van Spriel, A. B. Dynamic Plasma Membrane Organization: A Complex Symphony. Trends Cell Biol 2021, 31 (2), 119-129. https://doi.org/10.1016/j.tcb.2020.11.004.

(15) Popik, W.; Alce, T. M. CD4 Receptor Localized to Non-Raft Membrane Microdomains Supports HIV-1 Entry: IDENTIFICATION OF A NOVEL RAFT LOCALIZATION MARKER IN CD4 *. Journal of Biological Chemistry 2004, 279 (1), 704-712. https://doi.org/10.1074/jbc.M306380200.

(16) Stachowiak, J. C.; Schmid, E. M.; Ryan, C. J.; Ann, H. S.; Sasaki, D. Y.; Sherman, M. B.; Geissler, P. L.; Fletcher, D. A.; Hayden, C. C. Membrane Bending by ProteinProtein Crowding. Nat Cell Biol 2012, 14 (9), 944-949. https://doi.org/10.1038/ncb2561.

(17) Yuan, F.; Alimohamadi, H.; Bakka, B.; Trementozzi, A. N.; Day, K. J.; Fawzi, N. L.; Rangamani, P.; Stachowiak, J. C. Membrane Bending by Protein Phase Separation. PNAS 2021, 118 (11). https://doi.org/10.1073/pnas.2017435118.

(18) Banjade, S.; Rosen, M. K. Phase Transitions of Multivalent Proteins Can Promote Clustering of Membrane Receptors. eLife 2014, 3, e04123. https://doi.org/10.7554/eLife.04123.

(19) Zeno, W. F.; Johnson, K. E.; Sasaki, D. Y.; Risbud, S. H.; Longo, M. L. Dynamics of Crowding-Induced Mixing in Phase Separated Lipid Bilayers. J Phys Chem B 2016, 120 (43), 11180-11190. https://doi.org/10.1021/acs.jpcb.6b07119.

(20) Davis, S. J.; van der Merwe, P. A. The Kinetic-Segregation Model: TCR Triggering and Beyond. Nature immunology 2006, 7 (8), 803-809. https://doi.org/10.1038/ni1369.

(21) Varshney, P.; Yadav, V.; Saini, N. Lipid Rafts in Immune Signalling: Current Progress and Future Perspective. Immunology 2016, 149 (1), 13-24. https://doi.org/10.1111/imm.12617.

(22) Fujiwara, T.; Ritchie, K.; Murakoshi, H.; Jacobson, K.; Kusumi, A. Phospholipids Undergo Hop Diffusion in Compartmentalized Cell Membrane. Journal of Cell Biology 2002, 157 (6), 1071-1081. https://doi.org/10.1083/jcb.200202050.

(23) Wawrezinieck, L.; Rigneault, H.; Marguet, D.; Lenne, P. F. Fluorescence Correlation Spectroscopy Diffusion Laws to Probe the Submicron Cell Membrane Organization. 
Biophysical journal 2005, 89 (6), 4029-4042.

https://doi.org/10.1529/biophysj.105.067959.

(24) Lenne, P. F.; Wawrezinieck, L.; Conchonaud, F.; Wurtz, O.; Boned, A.; Guo, X. J.; Rigneault, H.; He, H. T.; Marguet, D. Dynamic Molecular Confinement in the Plasma Membrane by Microdomains and the Cytoskeleton Meshwork. The EMBO journal 2006, 25 (14), 3245-3256. https://doi.org/10.1038/sj.emboj.7601214.

(25) Eggeling, C.; Ringemann, C.; Medda, R.; Schwarzmann, G.; Sandhoff, K.; Polyakova, S.; Belov, V. N.; Hein, B.; von Middendorff, C.; Schonle, A.; Hell, S. W. Direct Observation of the Nanoscale Dynamics of Membrane Lipids in a Living Cell. Nature 2009, 457 (7233), 1159-1162. https://doi.org/10.1038/nature07596.

(26) Alenghat, F. J.; Golan, D. E. Membrane Protein Dynamics and Functional Implications in Mammalian Cells. Curr Top Membr 2013, 72, 89-120. https://doi.org/10.1016/B978-0-12-417027-8.00003-9.

(27) Schneider, F.; Waithe, D.; Clausen, M. P.; Galiani, S.; Koller, T.; Ozhan, G.; Eggeling, C.; Sezgin, E. Diffusion of Lipids and GPI-Anchored Proteins in Actin-Free Plasma Membrane Vesicles Measured by STED-FCS. Mol Biol Cell 2017, 28 (11), 1507-1518. https://doi.org/10.1091/mbc.E16-07-0536.

(28) Saffman, P. G.; Delbrück, M. Brownian Motion in Biological Membranes. PNAS 1975, 72 (8), 3111-3113. https://doi.org/10.1073/pnas.72.8.3111.

(29) Hughes, B. D.; Pailthorpe, B. A.; White, L. R. The Translational and Rotational Drag on a Cylinder Moving in a Membrane. Journal of Fluid Mechanics 1981, 110, 349372. https://doi.org/10.1017/S0022112081000785.

(30) Guigas, G.; Weiss, M. Size-Dependent Diffusion of Membrane Inclusions. Biophys $J$ 2006, 91 (7), 2393-2398. https://doi.org/10.1529/biophysj.106.087031.

(31) Frick, M.; Schmidt, K.; Nichols, B. J. Modulation of Lateral Diffusion in the Plasma Membrane by Protein Density. Current Biology 2007, 17 (5), 462-467. https://doi.org/10.1016/j.cub.2007.01.069.

(32) Petrov, E. P.; Schwille, P. Translational Diffusion in Lipid Membranes beyond the Saffman-Delbrück Approximation. Biophysical Journal 2008, 94 (5), L41-L43. https://doi.org/10.1529/biophysj.107.126565.

(33) Ramadurai, S.; Holt, A.; Krasnikov, V.; van den Bogaart, G.; Killian, J. A.; Poolman, B. Lateral Diffusion of Membrane Proteins. J. Am. Chem. Soc. 2009, 131 (35), 1265012656. https://doi.org/10.1021/ja902853g.

(34) Morozova, D.; Guigas, G.; Weiss, M. Dynamic Structure Formation of Peripheral Membrane Proteins. PLOS Computational Biology 2011, 7 (6), e1002067. https://doi.org/10.1371/journal.pcbi.1002067.

(35) Goose, J. E.; Sansom, M. S. P. Reduced Lateral Mobility of Lipids and Proteins in Crowded Membranes. PLOS Computational Biology 2013, 9 (4), e1003033. https://doi.org/10.1371/journal.pcbi.1003033.

(36) Wieser, S.; Moertelmaier, M.; Fuertbauer, E.; Stockinger, H.; Schütz, G. J. (Un)Confined Diffusion of CD59 in the Plasma Membrane Determined by HighResolution Single Molecule Microscopy. Biophysical Journal 2007, 92 (10), 37193728. https://doi.org/10.1529/biophysj.106.095398.

(37) Hartel, A. J. W.; Glogger, M.; Guigas, G.; Jones, N. G.; Fenz, S. F.; Weiss, M.; Engstler, M. The Molecular Size of the Extra-Membrane Domain Influences the Diffusion of the GPI-Anchored VSG on the Trypanosome Plasma Membrane. Sci Rep 2015, 5 (1), 10394. https://doi.org/10.1038/srep10394.

(38) Guigas, G.; Weiss, M. Membrane Protein Mobility Depends on the Length of ExtraMembrane Domains and on the Protein Concentration. Soft Matter 2015, 11 (1), 33-37. https://doi.org/10.1039/C4SM01846J. 
(39) Kim, D.-H.; Zhou, K.; Kim, D.-K.; Park, S.; Noh, J.; Kwon, Y.; Kim, D.; Song, N. W.; Lee, J.-B.; Suh, P.-G.; Lee, N. K.; Ryu, S. H. Analysis of Interactions between the Epidermal Growth Factor Receptor and Soluble Ligands on the Basis of SingleMolecule Diffusivity in the Membrane of Living Cells. Angewandte Chemie 2015, 127 (24), 7134-7138. https://doi.org/10.1002/ange.201500871.

(40) Yang, H.; Reinherz, E. L. Dynamic Recruitment of Human CD2 into Lipid Rafts. Linkage to T Cell Signal Transduction. J Biol Chem 2001, 276 (22), 18775-18785. https://doi.org/10.1074/jbc.M009852200.

(41) Yamabhai, M.; Anderson, R. G. W. Second Cysteine-Rich Region of Epidermal Growth Factor Receptor Contains Targeting Information for Caveolae/Rafts. J Biol Chem 2002, 277 (28), 24843-24846. https://doi.org/10.1074/jbc.C200277200. 